\title{
1 Einführung in die kulturvergleichende Psychiatrie
}

\author{
Thomas Stompe
}

In unserem Zeitalter der Globalisierung und der Kontinente übergreifenden Wanderungsbewegungen ist für Psychiater und Psychologen der Wissenserwerb über die Zusammenhänge zwischen psychischen Erkrankungen und Kultur eine durch die tagtägliche Arbeit mit Patienten mit Migrationshintergrund vorgegebene Notwendigkeit geworden. Im Rahmen von Auslandsbeschäftigungen ist man ebenfalls mit häufig fremdartig anmutenden Ausdrucksformen psychischen Krankseins konfrontiert, die ohne das nötige Fachwissen nicht verstanden werden können. Dieses Problem ist allerdings nicht neu. Bereits an der Wende vom 19. zum 20. Jahrhundert führten Imperialismus und Kolonialherrschaft zwangsläufig zu intensiven Begegnungen mit fremden Kulturen und damit zur Auseinandersetzung mit psychischen Krankheiten in außereuropäischen Regionen. In dieser Epoche ist daher die Geburtsstunde der Transkulturellen Psychiatrie anzusiedeln. Diese untersucht den Einfluss von Kultur und Gesellschaft auf die Existenz und Häufigkeit, den Verlauf, die Symptomgestaltung psychischer Krankheiten, sowie die Symptompräsentation und die Krankheits- und Behandlungskonzepte in verschiedenen Kulturen. Im Laufe des 20. Jahrhunderts entwickelten sich innerhalb der Transkulturellen Psychiatrie mit der kulturvergleichenden Psychiatrie, der Migrationspsychiatrie und der Ethnopsychiatrie drei Forschungs- und Arbeitsgebiete, die sich in ihrem Aufgabenfeld, ihrer Epistemiologie und Methodik in manchem unterscheiden (Stompe 2006; Ritter u. Stompe 2011).

Da der erste Band der Wiener Schriftenreihe für Transkulturelle Psychiatrie der Thematik der psychischen Erkrankungen im Kulturvergleich gewidmet ist, wird hier das komplexe Problemfeld der Pathogenese und Pathoplastik psychischer Erkrankungen unter Migrations- und Akkulturations- 
bedingungen ausgeklammert. Der Kulturvergleich selbst ist mit verschiedenen Problemen belastet (Srubal et al. 2005). Im Vorfeld der transkulturell-psychiatrischen Forschung gilt es einige wesentliche Punkte zu klären. Illustriert wird dies anhand des Beispiels der International Study on Psychotic Symptoms (ISPS), in der zwischen 1994 und 1999 in sieben Ländern (Georgien, Ghana, Litauen, Nigeria, Österreich, Pakistan und Polen) Daten von insgesamt 1.070 Patientinnen und Patienten mit Schizophrenie erfasst wurden.

\subsection{Epistemiologische Grundhaltung}

Innerhalb der transkulturellen Psychiatrie bildeten sich drei epistemiologische Vorannahmen über Krankheit und Kultur aus.

\subsubsection{Das partikularistische Krankheitsverständnis}

Von kulturanthropologisch orientierten Psychiatern wurde die Vergleichbarkeit der Manifestationsformen psychischer Erkrankungen aus Kulturen, die sich in ihren Menschen- und Weltbildern erheblich unterscheiden, grundsätzlich angezweifelt. Zu unterschiedlich und daher nahezu unübersetzbar seien die semantischen Felder, in welchen psychische Leidenszustände eingebettet sind, zu wenig vergleichbar die kulturspezifischen Krankheitskonzepte. Unter diesen Rahmenbedingungen könne die Suche nach universell gültigen Aussagen nur zu einer Nivellierung der Unterschiede führen. Die Kritik am partikularistischen Ansatz blieb von Seiten der universalistisch orientierten Psychiatrie allerdings nicht aus:

1. Ohne Referenzsystem sei es nicht möglich, überhaupt von einer bestimmten Erkrankung zu sprechen.

2. Was partikularistisch orientierte Forscher als kulturspezifische Erkrankung bezeichnen, könnte dem allgemein geteilten Krankheitsverständnis so fern stehen, dass man eigentlich schon von einem Culture-BoundSyndrom sprechen müsse.

\subsubsection{Das universalistische Krankheitsverständnis}

Die kulturvergleichende Psychiatrie geht zumeist von einem universalistischen Krankheitsverständnis aus. Universalistische Ansätze zielen auf die Herausarbeitung gemeinsamer Muster und Eigenschaften ab. Neben Universalien, die auf der allen Menschen gemeinsamen biologischen Ausstattung beruhen, können auch kulturelle Universalien beschrieben werden (Antweiler 2007). Die dem Universalismus verpflichteten Forscher halten daran fest, dass es ungeachtet aller kulturellen Unterschiede der Erscheinungsformen psychischer Erkrankungen, des zur Beschreibung psychischer Leidenszustände verwendeten semantischen Materials und der spezifischen Umgangsweise mit 
der Erkrankung, möglich sei, einen kulturinvarianten Kern herauszuarbeiten, der als Referenzpunkt für fremdethnische Manifestationsformen psychischer Erkrankungen dienen könne. Zu vermeiden gelte es allerdings, unkritisch die in der klassischen europäischen Psychiatrie als typisch angesehenen Beschreibungen als selbstverständliches Referenzsystem für andere Kulturen und Ethnien zu übernehmen. Das häufig nicht explizit dargelegte Ziel des kulturvergleichenden Ansatzes ist es dagegen, mit statistischen oder hermeneutischen Methoden Idealtypen klinischer Bilder herauszuarbeiten, die Nähe und Distanz dieser Idealtypen zu kulturspezifischen Phänotypen psychischer Erkrankungen darzustellen und damit eine validere Ausgangsbasis für epidemiologische Untersuchungen zu gewinnen.

\subsubsection{Das absolutistische Krankheitsverständnis}

Die innerhalb der psychiatrischen Epidemiologie verbreitete absolutistische Haltung geht davon aus, dass Krankheitsbilder in allen Kulturen gleich aussehen und demnach kontextfrei bestimmt werden können. Sie können daher empirisch leicht mit standardisierten Verfahren erfasst und ohne kulturspezifische Kompetenz gedeutet werden. Quantitative Vergleiche erscheinen unproblematisch und leicht interpretierbar. Aus terminologischen Gründen und wegen der Verwendung eines vergleichenden Ansatzes wird der Universalismus oft fälschlicherweise mit dem Absolutismus gleichgesetzt.

Für die ISPS wurde ein universalistischer Ansatz gewählt. Diese Zugangsweise ist deutlich aufwändiger als der absolutistische Ansatz.

\subsection{Wahl der Fragestellung}

Kulturvergleichende psychiatrische Studien zielen immer auf die Herausarbeitung von kulturunabhängigen Ähnlichkeiten und kulturgeprägten Unterschieden ab. Prinzipiell kann der Vergleich diachron (historisch) oder synchron (im Querschnitt) erfolgen. In der Folge wollen wir uns ausschließlich mit synchronen Untersuchungsdesigns auseinandersetzen. Soll in einer populationsbasierten Studie das gesamte mögliche Spektrum an Erkrankungen verglichen werden, beschränkt man sich auf die Untersuchung der Häufigkeit einer einzelnen Erkrankung oder auf die Häufigkeit einzelner Symptome einer bestimmten Krankheit? Je breiter das Spektrum der erfassten Erkrankungen ist, desto geeigneter ist ein absolutistischer Ansatz, je enger die Fragestellung, desto günstiger ist ein universalistischer Zugang. Neben Krankheiten und deren Symptomatik können aber auch Haltungen zu Krankheiten und Störungsbildern (Krankheitskonzepte, Stigmatisierung, familiäre und soziale Unterstützung etc.), Familiensystemen und v.a.m. untersucht werden (s. Tab. 1). 
1 Einführung in die kulturvergleichende Psychiatrie

Tab. 1 Untersuchungsschwerpunkte in der kulturvergleichenden Psychiatrie

\begin{tabular}{llr}
$\begin{array}{l}\text { Schwerpunkt } \\
\text { Krankheit, klinisches Bild }\end{array}$ & Parameter & $\begin{array}{c}\text { epistemiologischer Zugang } \\
\text { absolutistisch, universalistisch }\end{array}$ \\
\cline { 2 - 3 } & Subtypen, Syndrome (quantitativ) & absolutistisch, universalistisch \\
\hline & Verlauf, Ausgang & absolutistisch \\
\hline Syndrome, Symptome & Phänomenologie & universalistisch \\
\hline Behandlung & Setting, Ansprechen, Nebenwirkungen & absolutistisch \\
\hline Krankheitskonzepte & $\begin{array}{l}\text { Einzelitems, übergeordnete Erklärungs- } \\
\text { muster }\end{array}$ & universalistisch \\
\hline Stigmatisierung & Einzelitems, übergeordnete Bereiche & universalistisch \\
\hline soziale Unterstützung & Einzelitems, übergeordnete Bereiche & universalistisch \\
\hline Familienstrukturen & $\begin{array}{l}\text { Familienformen, Mitgliederanzahl, } \\
\text { Familienstand der Eltern, Geschwister- } \\
\text { reihen etc. }\end{array}$ & absolutistisch \\
\hline soziale Integration & $\begin{array}{l}\text { Familienstand, Ausbildung, Beruf } \\
\text { des Patienten }\end{array}$ & absolutistisch \\
\hline
\end{tabular}

Die Ergebnisse können entweder für sich analysiert (Ritter et al. 2010; Stompe et al. 2010) oder mit Daten zur Häufigkeit, Verlauf und Gestaltung der Symptomatik einer psychischen Erkrankung in Beziehung gesetzt werden.

\subsection{Referenzsysteme}

Bevor man die Wirkungsweise lokaler Faktoren auf die Entstehung und Gestaltung psychischer Krankheiten studiert, muss festgelegt werden, welche meso- bzw. makrosoziokulturellen Einheiten für den Vergleich herangezogen werden und wie diese definiert sind (s. Tab. 2).

Tab. 2 Spektrum der Referenzgruppen

\begin{tabular}{|c|c|c|}
\hline Einheiten & Anzahl & Bemerkungen \\
\hline Menschen (Individuen) & 6,56 Mrd. & $\begin{array}{l}\text { = ca. } 5,8 \% \text { aller je gelebten Menschen } \\
\text { (106,4 Mrd., seit } 50.000 \text { v.u.Z.) }\end{array}$ \\
\hline $\begin{array}{l}\text { Sprachen, linguistische } \\
\text { Gruppen }\end{array}$ & $5.000-7.000$ & $\begin{array}{l}\text { starke Abnahme der Zahl: derzeit schon etwa } \\
2.000 \text { Sprachen mit weniger als } 1.000 \text { Sprechern }\end{array}$ \\
\hline $\begin{array}{l}\text { Ethnien: „Wir“-Kollektiv mit } \\
\text { spezifischer Kulturtradition }\end{array}$ & $2.500-8.000$ & $\begin{array}{l}\text { Spektrum reicht von Ethnien mit wenigen Mitgliedern } \\
\text { bis ca. 1. Mrd. (Han-Chinesen) }\end{array}$ \\
\hline Staaten & 225 & $\begin{array}{l}\text { Zunahme (1972: } 172 \text { Staaten; aber } 1000 \text { v. Chr. } \\
\text { ca. } 20.000 \text { politische Einheiten) }\end{array}$ \\
\hline Kulturregionen, „Kulturkreise“ & $9-20$ & 9 Zivilisationen nach Huntington \\
\hline Menschheit & 1 & Homo sapiens \\
\hline
\end{tabular}


Im angloamerikanischen Raum werden häufig Rassen verglichen (Kaukasier, Afroamerikaner, Latinos etc.). Im Gegensatz zum Rassenbegriff der deutschen Sprache, ist race im Englischen ein Terminus, der biologisch und kulturell konnotiert ist. In der deutschsprachigen Transkulturellen Psychiatrie sind die verglichenen Einheiten zumeist Staaten oder Großregionen, manchmal auch Sprachgruppen, Ethnien oder Kulturkreise.

Staaten sind politische Gebilde, die ethnisch und sprachlich in unterschiedlichem Maße homogen sind. Während etwa die eingesessene österreichische Bevölkerung sprachlich und ethnisch ziemlich homogen ist, gibt es bereits in der Schweiz vier gleichberechtigte Sprachen und in Nigeria sind sogar 511 lebende Sprachgemeinschaften katalogisiert. Insgesamt ist die angenommene Zahl der Sprachen rückläufig (geschätzt zwischen 5.0oo und 7.00o).

Ethnie oder Ethnos (von griechisch ع̋قvos, éthnos, „Volk, Volkszugehörige“) ist ein Begriff aus der Sozial- und Kulturanthropologie (früher Ethnologie oder Völkerkunde). Ethnologen fassen mit diesem Begriff benannte Populationen von Menschen zusammen, die Herkunftssagen, Geschichte, Kultur, Verbindung zu einem spezifischen Territorium und ein Gefühl der Solidarität miteinander teilen. Durch den Begriff „ethnisch“ werden Menschen nicht nach linguistischen Gesichtspunkten in Gruppen eingeteilt. In manchen Ländern (vor allem in Einwanderungsländern wie den USA oder Kanada) benutzen verschiedene ethnische Gruppen dieselbe Sprache als gemeinsame Verkehrssprache. Andererseits gibt es innerhalb der Sprachen vieler ethnischer Gruppen stark ausgeprägte Dialekte, die sich im Laufe der Zeit in verschiedene Sprachen auseinander entwickeln können.

Großregion ist ein geographischer Begriff, der Bevölkerungsgruppen umfasst, die gewisse sprachliche oder kulturelle Gemeinsamkeiten zeigen (etwa „Westafrika“ oder „Indochina“). Wie leicht ersichtlich, sind diese Einheiten nicht deckungsgleich mit dem Kulturbegriff.

Der Terminus Kultur kommt vom Lateinischen cultura was soviel meint wie „Bearbeitung“, „Pflege“, „Ackerbau“, von colere, „wohnen“, „pflegen“, „den Acker bestellen“. Für den Systemtheoretiker Niklas Luhmann (1999) beginnt Kultur geschichtlich gesehen erst dann, wenn es einer Gesellschaft gelingt, nicht nur Beobachtungen vom Menschen und dessen Umwelt anzustellen, sondern auch spezielle Formen und Blickwinkel der Beobachtungen der Beobachtungen zu entwickeln. Diese Voraussetzung wurde an der Schwelle zur Moderne Ende des 18. Jahrhunderts erreicht. Seither wird der Terminus in der heute gebräuchlichen Weise verwendet. Er erfuhr zahlreiche Begriffsbestimmungen, die unterschiedliche Bedeutungsfacetten fokussierten. Eine Definition, die für die Transkulturelle Psychiatrie ausreichend weit ist, stammt von Alexander Thomas:

„Die Kultur einer Gruppe oder Klasse umfasst die besondere und distinkte Lebensweise dieser Cruppe oder Klasse, die Bedeutungen, Werte und Ideen, wie sie in Institutionen, in 
den gesellschaftlichen Beziehungen, in den Claubenssystemen, in Sitten und Bräuchen, im Gebrauch der Objekte und im materiellen Leben verkörpert sind. Kultur ist die besondere Gestalt, in der dieses Material und diese gesellschaftliche Organisation des Lebens Ausdruck findet. Eine Kultur enthält die ,Landkarten der Bedeutung', welche Dinge für ihre Mitglieder verstehbar machen“(Thomas et al. 2009).

Damit wird eine wichtige, auch von den Bielefelder Systemtheoretikern immer wieder hervorgehobene Funktion verdeutlicht: In einer komplexen natürlichen und sozialen Umwelt helfen kulturelle Muster Kontingenz zu reduzieren, was vermutlich ein evolutionärer Vorteil ist.

Als Kulturkreis oder Kulturraum bezeichnet man ein großflächiges Siedlungsgebiet, dessen Mitgliedern die gleiche oder zumindest eine ähnliche Kultur zugeschrieben wird. Das Konzept des Kulturkreises übersteigt in seiner Reichweite meist andere Einteilungen sozialer Einheiten wie soziale Gruppen, Stämme, Gesellschaften, Nationen oder „Völker“. So spricht man beispielsweise vom „christlichen Abendland“ bzw. vom „islamischen Orient“ als zwei großen Kulturkreisen, die sich durch bestimmte Wertvorstellungen, soziale Normen, Sitten und Gebräuche auszeichnen. Wichtige klassische Vertreter der Kulturkreistheorie waren Leo Frobenius, Oswald Spengler und Arnold Toynbee. Die Definition der Größe eines Kulturkreises wird unterschiedlich vorgenommen und richtet sich nach der Auswahl von Bestimmungen, die für die Unterscheidungen typisch sein sollen. So wird einerseits von einem skandinavischen Kulturkreis gesprochen, der anderseits auch Teil des europäischen Kulturkreises sein kann. Eine beliebte Einteilung ist die in „Westlicher Kulturkreis“, „Fernöstlicher Kulturkreis“ oder in „Arabischer Kulturkreis“. Wichtige lebende Vertreter einer gemäßigten Fassung dieser Theorie sind Samuel Huntington oder Bassam Tibi.

Welches Referenzsystem verwendet wird, hängt letztlich vom untersuchten Kollektiv und den zur Erklärung anstehenden Ergebnissen ab. Jeder Mensch ist Teil einer sprachlichen und kulturellen Gemeinschaft, die wieder zu größeren Einheiten zusammengefasst werden kann. In jedem Fall sollte man sich aber über Reichweite und Grenzen der verwendeten Begriffe im Klaren sein. In der IPSP erhoben wir die ethnische Zugehörigkeit und die Staatsangehörigkeit. Da bei kulturvergleichenden psychiatrischen Studien Migrationshintergrund ein Ausschlusskriterium darstellt, stimmen in den Österreich, Polen und Litauen Staatsangehörigkeit und ethnische Zugehörigkeit überein. Ganz anders ist die Situation jedoch in Georgien, Pakistan, Nigeria und Ghana zu bewerten, wo verschiedene ethnische Gruppierungen und Sprachgemeinschaften leben. Dies sei am Beispiel der beiden Westafrikanischen Staaten illustriert (s. Abb. 1).

Diskriminanzanalytisch zeigte sich, dass sich die Mitglieder der einzelnen Ethnien in ihrer produktiv-psychotischen Symptomatik in weniger als 10\% der Varianz unterschieden, ähnliches ergab der Vergleich zwischen den beiden 
1.4 Auswahl der teilnehmenden Zentren, der Mitarbeiter/-innen, der Untersuchungsinstrumente, Ratertraining

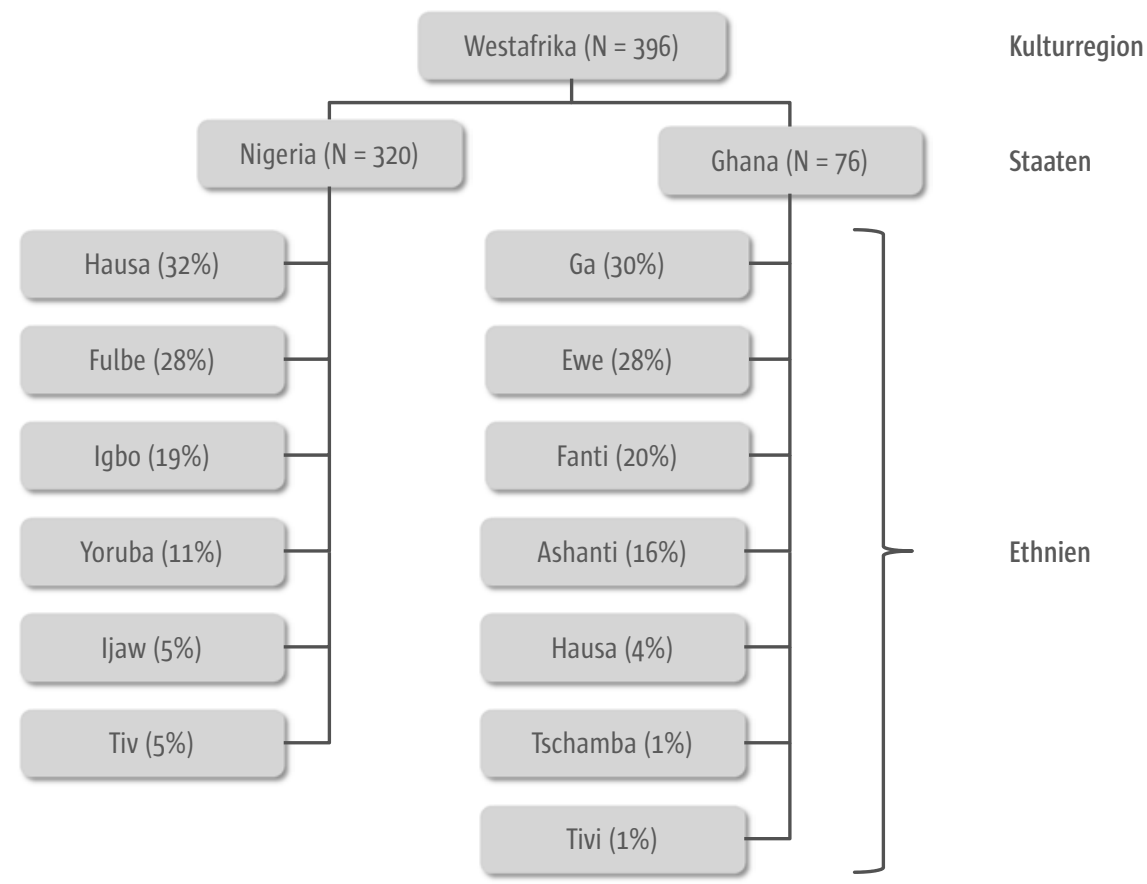

Abb. 1 Afrikanische Patienten der International Study of Psychotic Symptoms nach ethnischer Zugehörigkeit, Staatsbürgerschaft und Kulturregion

Westafrikanischen Staaten. Es kann also postuliert werden, dass sich gewisse Eigenheiten des Westafrikanischen Kulturraums (Beispiele wären der Claube an Geister, Magie und Zauberei, ähnliche familiäre Sozialisationsbedingungen) vereinheitlichend auf die Phänomenologie psychotischer Symptome auswirken.

\subsection{Auswahl der teilnehmenden Zentren, der Mitarbeiter/-innen, der Untersuchungsinstrumente, Ratertraining}

Da, wie oben erwähnt, die kulturvergleichende Psychiatrie auf das Auffinden von kulturunabhängigen Ähnlichkeiten und kulturbedingten Unterschieden ausgerichtet ist, bewährt es sich für gewöhnlich, Länder oder Ethnien in die Studie einzuschließen, die eine ausreichende kulturelle Distanz zueinander aufweisen. Die Mitarbeiter der beteiligten Zentren sollten ausreichende Erfahrungen mit den kulturspezifischen Krankheitskonzepten und Symptomen besitzen. Es hat sich bewährt, sich auf Fachleute zu stützen, die einen Teil ihrer Ausbildung in Europa oder Nordamerika absolviert haben und bereits wissenschaftlich gearbeitet haben. Jede lokale Studiengruppe sollte aus zwei Ratern bestehen. 
Die Auswahl der Untersuchungsinstrumente erfolgt nach drei Gesichtspunkten:

- Fragestellungen

- Vorliegen von geeigneten Instrumenten in den jeweilige Landessprachen

- epistemiologischer Ausgangspunkt

Im Rahmen der ISPS musste für die Erfassung der psychotischen Symptomatik schizophrener Erkrankungen ein eigenes Instrument entwickelt werden, da die bereits vorhandenen Fragebögen entweder von einem absolutistischen Krankheitsverständnis ausgingen oder für unsere Fragestellungen zu undifferenziert waren. Anhand der klassischen psychopathologischen Literatur und der vorliegenden transkulturell-psychiatrischen Arbeiten wurde eine Sammlung psychotischer Phänomene angelegt. Für die relevanten Zielitems wie Wahn oder Halluzinationen wurden aus der Literatur Definitionen gewählt, die in allen teilnehmenden Zentren akzeptiert wurden (Stompe 2008, 2010; Bauer et al. 2011). Darüber hinaus gibt es in dem eigens dafür entwickelten Fragebogen für psychotische Symptome neben den dichotom zu beantwortenden Items ausführlich Raum für die semistrukturierte deskriptive Beschreibung der Symptomatik. Wenn ein Patient das Vorliegen eines Symptoms bejahte, wurde er gebeten, einige Beispiele dafür zu geben. Wenn er etwa angab, Stimmen zu hören, wurde nach dem Thema, der Lokalisation, der Häufigkeit, der Dauer usw. nachgefragt. Damit wurde die Datenerhebung deutlich umfassender. Dadurch wurde es erst möglich, Stimmen mit großer Sicherheit von Gedankenlautwerden, Gedankeneingebungen, illusionären Verkennungen oder Vorstellungskonkretisierungen abzugrenzen (Stompe et al. 2003; Bauer et al. 2011)

Der strukturierte Abschnitt der deutschen Version des Fragebogens wurde einerseits mit dem von uns verwendeten diagnostischen Manual (SCID 1), andererseits mit dem semistrukturierten deskriptiven Abschnitt des Fragebogens validiert. Das Instrument wurde von den beiden österreichischen Ratern auf Interrater- und Retest-Reliabilität geprüft. Als nächster Schritt wurde der Bogen in die jeweiligen Landesprachen übersetzt und rückübersetzt. Die Übersetzung erfolgte im jeweiligen Zentrum, die Rückübersetzung in Österreich. Am aufwendigsten gestaltete sich das Rater-Training. Dabei wurde wie folgt vorgegangen: In jedem Zentrum wurden von einem Rater fünf aufwändige Kasuistiken zusammengestellt und an alle übrigen beteiligten Länder verschickt. Bereits zuvor wurden die Fallberichte von dem zweiten Rater beurteilt. Anschließend wurden alle Kasuistiken von sämtlichen Studienteilnehmern unter Zuhilfenahme des Fragebogens ausgewertet. Die Ergebnisse wurden geprüft, abweichende Bewertungen wurden solange diskutiert, bis Einigung bestand. Dieser Vorgang wurde ein halbes Jahr lang regelmäßig wiederholt, bis eine ausreichend gute Interrater-Reliabilität vorlag. Das Training erfolgte über Internet. 


\subsection{Interpretation der Ergebnisse}

Im Falle statistisch signifikanter Ergebnisse gibt es drei sich ergänzende Ansätze, um psychopathologische mit soziokulturellen Daten in Verbindung zu setzen:

- Zur hermeneutischen Interpretation der Ergebnisse können soziologische, kulturpsychologische, kulturanthropologische, kulturgeschichtliche, kulturphilosophische oder religionsgeschichtliche Arbeiten über die jeweiligen Ethnien, Staaten oder Kulturregionen herangezogen werden.

- Auf der makrosozialen Ebene können demographische Daten wie der Modernisierungsgrad, die gesellschaftliche Ungleichheit, der Grad der Demokratisierung, die Verteilung der religiösen Konfessionen und andere Parameter der beteiligten Regionen zur Interpretation der Ergebnisse herangezogen werden. Diese aggregierten Daten können mit Prävalenzen korreliert werden. Die makrosozialen Parameter beziehen sich zwar nicht direkt auf die individuelle Situation des Patienten, beschreiben aber das größere Umfeld in dem das Individuum aufgewachsen ist und lebt. Auch wenn sich die individuellen Wertehaltungen des Einzelnen von denen der Mehrheitsgesellschaften unterscheiden, kann dieser sich üblicherweise nicht gänzlich dem Einfluss des gesellschaftlichen Umfelds entziehen.

- Auf der meso- und mikrosozialen Ebene werden individualisierte Daten, wie das persönliche religiöse Bekenntnis, verschiedenste Wertehaltungen, Sozialisationsparameter, Daten zum kulturellen Milieu und ähnliches mehr, mit psychopathologischen Daten in Verbindung gesetzt. Während durch makrosoziale Daten eher implizite Einflüsse von Kultur und Gesellschaft auf psychische Parameter erfasst werden, beschreiben mikro- und mesosoziale Faktoren den Einfluss der unmittelbaren Lebenswelt. Idealerweise sollten alle drei Methoden zur Interpretation der Ergebnisse kulturvergleichender Untersuchungen herangezogen werden.

Wie in den Beiträgen dieses Bandes ausführlich beschrieben wird, gibt es deutliche interkulturelle Variationen in der Häufigkeit und Gestaltung psychischer Erkrankungen. Selbst bei einer Störung mit ausgeprägter Heritabilität wie der Schizophrenie, von der man ursprünglich annahm, dass sie in allen Teilen der Welt in ähnlicher Verteilung vorkommt, konnten neue Metaanalysen Schwankungen der Prävalenz und Inzidenz nachweisen (McGrath et al. 2004; Saha et al. 2005). Dabei scheinen unterschiedliche kulturelle und biologische Faktoren für die Verteilungsunterschiede und für die phänomenologische Gestaltung psychischer Erkrankungen verantwortlich zu sein, was im Folgenden dargestellt werden soll. 


\subsection{Kultur und Pathogenese psychischer Erkrankungen}

Nicht nur schizophrene Störungen, sondern die meisten bekannten psychischen Erkrankungen zeigen beträchtliche regionale Häufigkeitsschwankungen. Ein zentrales Aufgabenfeld der kulturvergleichenden Psychiatrie ist die systematische Suche nach den dafür verantwortlichen Faktoren. Dafür verwendet sie das methodische Rüstzeug von Nachbarwissenschaften wie der psychiatrischen Epidemiologie, der Kultur- und Sozialanthropologie sowie der Kultur- und Religionswissenschaften (Ritter u. Stompe 2011). Da es in den letzten 10 Jahren in der Transkulturellen Psychiatrie zu einer Annäherung zwischen kulturwissenschaftlichen und biologischen Ansätzen kam, werden immer häufiger populationsgenetische Theorien in die Überlegungen miteinbezogen.

Prinzipiell ist davon auszugehen, dass viele der ätiologischen und pathogenetischen Konstellationen, die in der westlichen Psychiatrie in den letzten 100 Jahren herausgearbeitet wurden, auch in anderen Kulturen Gültigkeit haben. Darüber hinaus gilt es allerdings Häufigkeitsunterschiede zu erklären, die auf teilweise noch unbekannte, entweder pathogen und protektiv wirksame, kulturspezifische oder regionale biologische Varianten zurückgeführt werden müssen. Abbildung 2 zeigt ein heuristisches Modell, das den Rahmen für die Integration der (künftigen) Ergebnisse der verschiedensten Fachrichtungen bilden könnte.

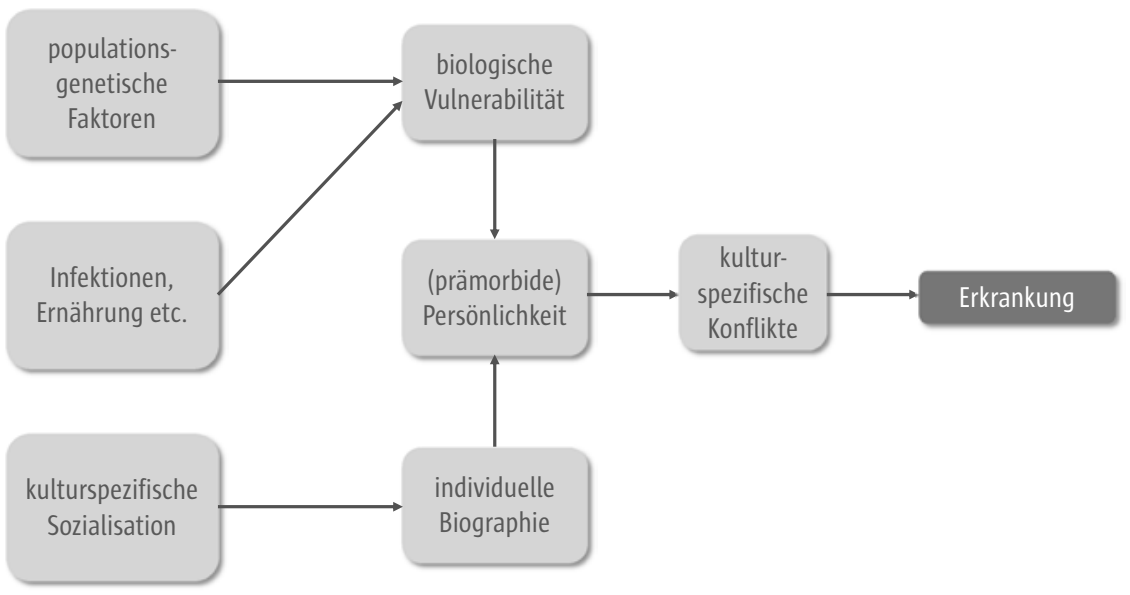

Abb. 2 Modell des Einflusses von Kultur und spezifischen biologischen Faktoren auf die Pathogenese psychischer Erkrankungen

Wie vor allem das junge Fach der Ethnopsychopharmakologie zeigt, gibt es pharmakogenetische Varianten, die sich auf die Metabolisierung und damit auf Wirkung und Nebenwirkungen von Psychopharmaka auswirken (Lin et al. 2008). Diese Erkenntnis ist nicht weiter überraschend; bereits in den 199oer- 
Jahren konnten Cavalli-Svorza et al. (1994) populationsspezifische genetische Landkarten erstellen, die vorwiegend durch Gendrift und Wanderungsbewegungen erklärbar sind. In der Zwischenzeit liegt für nahezu jede Ethnie eine Fülle populationsgenetischer und molekulargenetischer Untersuchungen vor, die die Ethnogenese präzise nachvollziehbar machen. Es kann daher nicht ausgeschlossen werden, dass ein Teil der Prävalenzunterschiede auf genetischen Variationen beruht. Da zu den meisten psychischen Erkrankungen bisher keine konkreten molekulargenetischen Ergebnisse vorliegen, muss von einer uneinheitlichen polygenetischen Heritabilität ausgegangen werden. Wie Untersuchungen in Isolaten gezeigt haben, ist es schwierig, lokale genetische Varianten herauszufiltern (Baluyeva et al. 2007).

Auch nicht-genetische Faktoren wie lokale Ernährungsgewohnheiten, exotische (Schwangerschafts-)Infektionen sowie häufige Schwangerschafts- oder Geburtskomplikationen können einen Beitrag zu regionalen Prävalenzschwankungen leisten (z.B.: Mackay-Sim et al. 2004). Erwähnt werden soll an dieser Stelle die Hypothese von Torrey (2001), der davon ausgeht, dass der Anstieg der Prävalenz der Schizophrenie in Europa und Nordamerika auf die stille Ausbreitung einer Slow-virus Infektion zurückzuführen ist. Daraus würde sich auch das seltene Vorkommen dieser Erkrankung in abgelegenen Stammesgesellschaften ableiten lassen. Diese regional- und kulturspezifischen biologischen Faktoren können zusammen mit allgemeinen biologischen Faktoren zur Gesamtvulnerabilität beitragen.

Obwohl biologische Forschungsergebnisse zunehmend Gewicht für die pathogenetischen Modelle der Transkulturellen Psychiatrie bekommen, liegt der Forschungsschwerpunkt auf dem Einfluss der kulturellen Sozialisationsbedingungen auf Entstehung und Häufigkeit psychiatrischer Erkrankungen. Die Ethnopsychoanalyse und die Kulturpsychologie etwa zeigten, wie sich gesellschaftliche Wertehaltungen, vermittelt über die Familie und die Gemeinschaft, auf die Entwicklung von Persönlichkeitseigenschaften auswirken. Parin et al. (1963) wiesen bei dem in Nigeria lebenden Stamm der Dogon kollektive Ich- und Über-Ich-Strukturen nach, die als Gruppen-Ich und KlanGewissen bezeichnet wurden. Der Einfluss der Kultur reicht weit in Wahrnehmungsverarbeitung, Urteilsbildung und die Gestaltung der Identität hinein (Kitayama u. Cohen 2007). Die kulturvergleichende Gehirnforschung konnte sogar nachweisen, dass diese kulturspezifischen psychologischen Merkmale teilweise mit der Aktivierung unterschiedlicher neuronaler Netzwerke im Gehirn assoziiert sind (Chiao 2009). Inzwischen hat die Erforschung des Einflusses von kulturspezifischen psychologischen und neurobiologischen Eigenschaften auf die Psychopathologie begonnen (Choudhury u. Kirmayer 2009).

All diese biologischen und soziokulturellen Faktoren tragen neben der individuellen biographischen Entwicklung zur Formung der Persönlichkeit bei. Jeder Mensch verfügt aufgrund seiner ontogenetischen Entwicklung über ein mehr oder weniger gut ausgebildetes Potenzial, alltägliche und nichtalltäg- 
liche Belastungen zu bewältigen. Es gibt allerdings kulturspezifische Belastungen und Konflikte, die bei vulnerablen Menschen psychische Erkrankungen auslösen können. Frauen in patrilinearen und patriarchalischen Gesellschaften heiraten für gewöhnlich in sehr jungen Jahren in die Familie des Ehemannes ein (Patrilokalität). Sie unterstehen der Herrschaft ihrer Schwiegermütter und haben mit einem Schlag die soziale Unterstützung der Herkunftsfamilie verloren. Häufige Folge sind psychosomatische, dissoziative oder affektive Erkrankungen. Aber auch im westlichen Kulturraum finden sich spezifische Belastungszonen, die zu Persönlichkeits- und Verhaltensauffälligkeiten sowie psychischen Störungen führen können. Die männliche Identitätsfindung unter den Bedingungen des Zusammenbruchs allgemein gültiger hegemonialer Männlichkeitsbilder im Zeitalter der Postmoderne führt bei prädisponierten Jugendlichen zu narzisstischen und dissozialen Persönlichkeitsentwicklungen und zu gewalttätigen Verhaltensweisen (Robertz u. Wickenhäuser 2010). Bei Frauen sind westliche weibliche Schönheitsideale mit dem häufigen Auftreten von Anorexia nervosa und Bulimie assoziiert. Als Folge subjektiver Arbeitsüberlastung ist das Burn-out Syndrom in spätkapitalistischen Gesellschaften geradezu zu einem Leistungsnachweis geworden. Starre soziale Rollenübernahmen können bei Frauen in Europa zu Depressionen (Tellenbach 1960), in Malaysia zu Latah, einem dissoziativen kulturspezifischen Syndrom, führen (Pfeiffer 1994).

\subsection{Kultur und die Gestaltung psychischer Erkrankungen}

Während die regionalen Schwankungen der Häufigkeit psychischer Erkrankungen vermutlich auf komplexe Wechselwirkungen biologischer und soziokultureller Faktoren zurückgehen, ist der Einfluss der Kultur vorwiegend für die lokalen Unterschiede der phänomenologischen Gestaltung der Symptomatik verantwortlich (s. Abb. 3).

Die durch Sozialisationsinstanzen wie Familie, Peergroup, Schule und Massenmedien an jeden Einzelnen vermittelten Werte und Symbolsysteme beeinflussen zusammen mit krankheitsspezifischen Faktoren die Ausgestaltung der Symptomatik. Die angeführten Sozialisationsinstanzen sind integraler Teil der Lebenswelt des Patienten (Schütz u. Luckmann 1979). Diese ist die „ausgezeichnete Wirklichkeit“, in der jeder Mensch lebt, denkt, handelt und sich mit anderen verständigt. Die Alltagswelt ist vorgegeben und wird fraglos und selbstverständlich hingenommen, sie ist der unhinterfragte Boden aller Geschehnisse. Sie ist von Anfang an eine intersubjektive Kulturwelt, in der alle Tatsachen immer schon interpretierte Tatsachen sind, die auf Sinnzusammenhänge und Deutungsmuster verweisen, welche Erfahrung und Handeln in der alltäglichen Welt ermöglichen. Sobald sich bei Kindern das Symbolverständnis entwickelt hat, werden über die Sozialisationsinstanzen der Lebenswelt Werte und Symbolsysteme vermittelt, die gemeinsam mit individuellen 


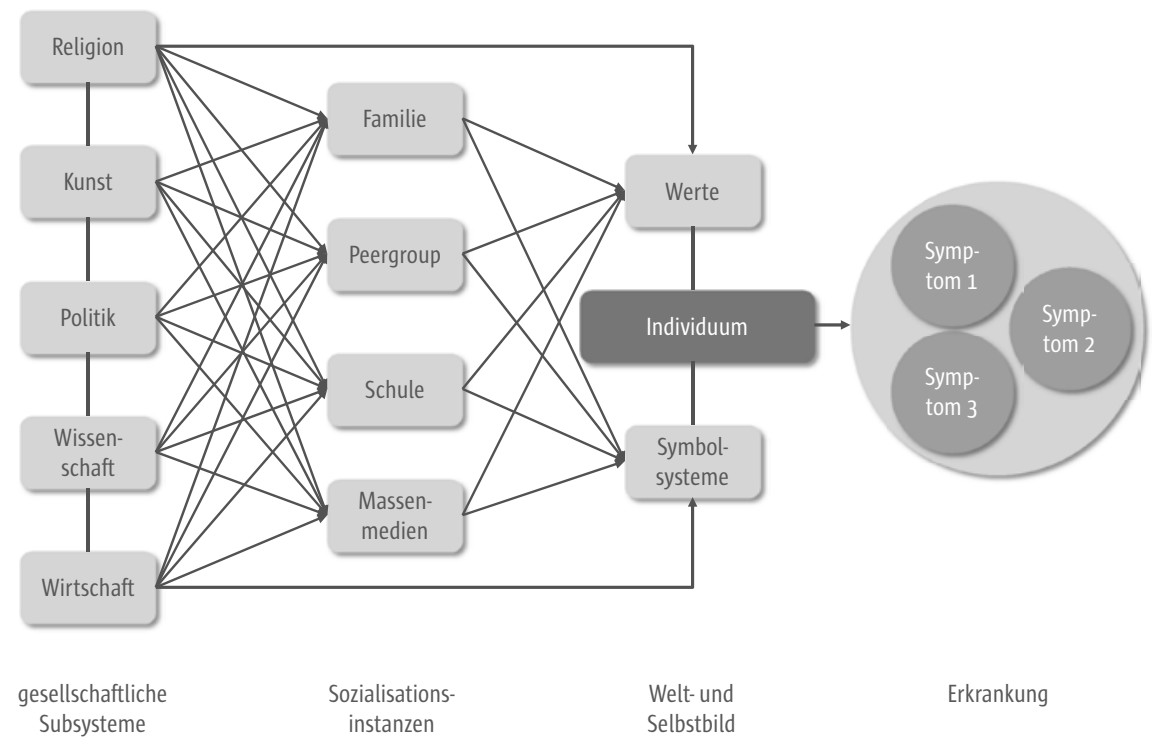

Abb. 3 Modell des Einflusses der Kultur auf die Symptomgestaltung psychischer Erkrankungen

biographischen Erfahrungen das Weltbild des Einzelnen formen. Diese von Familie, Peergroup, Schule und Massenmedien vermittelten Werte und Symbole sind wiederum durch die Semantik gesellschaftlicher Subsysteme wie Religion, Moral, Wirtschaft, Wissenschaft, Kunst, Recht oder Politik generiert (Luhmann 1993-1999). Die Semantik gesellschaftlicher Subsysteme unterliegt einem historischen Wandel, durch den sich ein Teil der Veränderungen schizophrener Wahnthemen in den letzten 150 Jahren erklären lässt (Stompe et al. 2003).

Wenn man sich der Bedeutung der lokalen Unterschiede von Struktur und Inhalt psychischer Symptome und Syndrome über die hermeneutische Analyse der Semantik gesellschaftlicher Subsysteme anzunähern versucht, ergeben sich einige methodische Probleme. Die Gesellschaft unterliegt nach Luhmann einer evolutionären Entwicklung, die von segmental differenzierten tribalen Gemeinschaften über die Zentrum-Peripherie-Differenzierung der frühen Hochkulturen zu den hierarchisch differenzierten Gesellschaften verlaufen ist. Erst in der Neuzeit begann sich die in der (post-)industriellen Welt dominante funktionale Differenzierung herauszubilden (Luhmann 1998). Voll entwickelt, sind in funktional differenzierten Gesellschaften soziale Subsysteme voneinander unabhängig und nur strukturell miteinander gekoppelt. Keines der Subsysteme ist dem anderen übergeordnet. Die Wirtschaft etwa ist ebenso von der Wissenschaft abhängig wie umgekehrt, das Rechtssystem und die Politik sind selbstverständlich strukturell gekoppelt, arbeiten aber unabhängig voneinander (s. Abb. 4). 


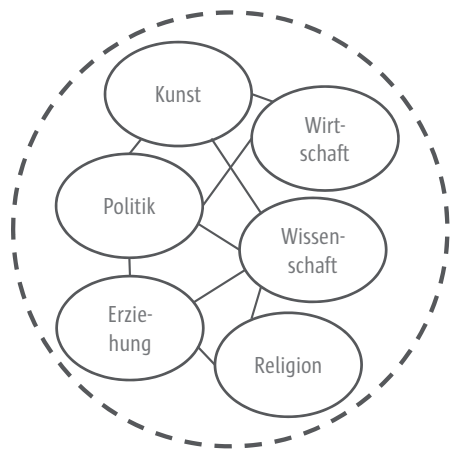

Funktionale Differenzierung

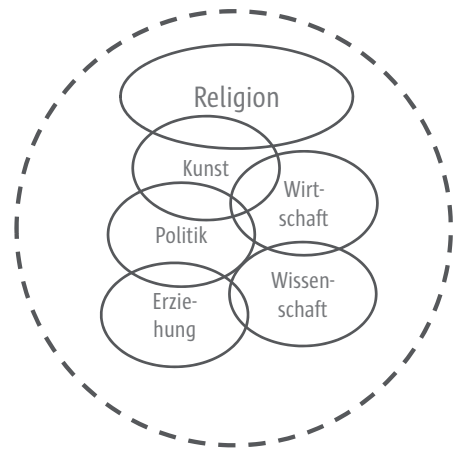

Hierarchische Differenzierung

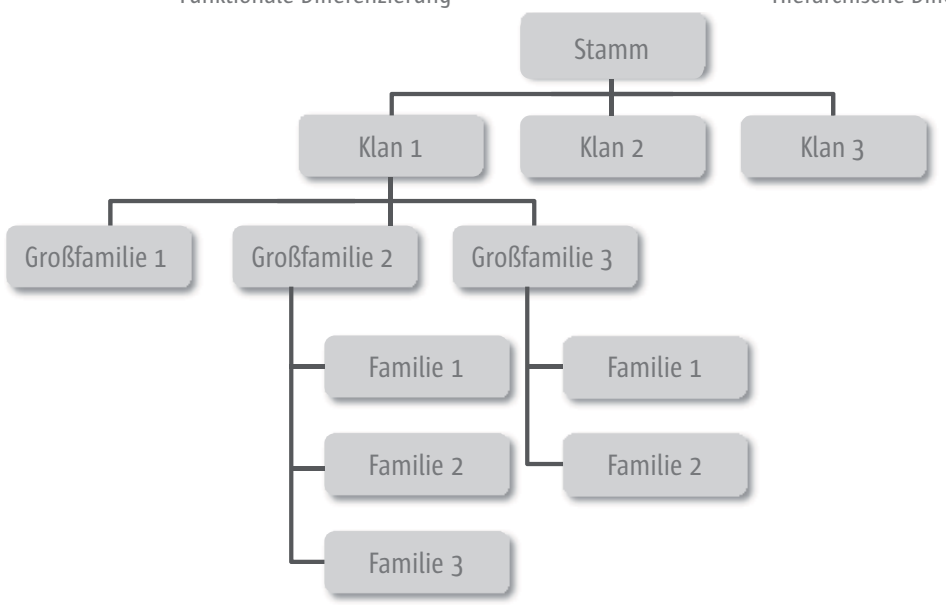

Segmentale Differenzierung

Abb. 4 Funktional, hierarchisch und segmental differenzierte Gesellschaften

Ganz anders ist die Lage in hierarchisch differenzierten Gesellschaften. Die einzelnen Subsysteme sind in wesentlich geringerem Maße voneinander unabhängig, eines, zumeist die Religion, beansprucht die Führungsrolle. Wiederum anders ist das in segmental differenzierten Stammesgesellschaften, in denen sich spezialisierte gesellschaftliche Subsysteme noch gar nicht entwickeln konnten. Wenn man also etwa den Einfluss der Religion auf Häufigkeit und Inhalt des religiösen Wahns in Österreich, Pakistan oder in einem Yoruba-Dorf in Nigeria analysieren möchte, gilt es zu beachten, dass Religion in jeder dieser Sozietäten einen vollkommen andersartigen Bedeutungsumfang hat und gänzlich unterschiedlich verankert ist. Kompliziert wird die Situation darüber hinaus durch den Umstand, dass im Zuge der Kolonialisierung und später der Globalisierung einzelne Stadien der gesellschaftlichen Entwicklung übersprungen werden und Hybridgesellschaften entstanden sind. In Nigeria und anderen afrikanischen Staaten etwa werden traditionelle segmentale Stammesstrukturen durch hierarchische Stratifizierungen und funktionell differenzierte Subsysteme überlagert. 
Diese Einführung in die Fragestellungen, methodischen Probleme und die Aussagekraft der kulturvergleichenden Psychiatrie sollte ermuntern (und nicht abschrecken), sich mit diesem komplexen Forschungsgebiet auseinanderzusetzen. Die Beiträge dieses Bandes präsentieren Ergebnisse der Forschungsanstrengungen der kulturvergleichenden Psychiatrie zum Einfluss der Kultur auf Häufigkeit und Gestaltung klinischer Krankheitsbilder.

\section{Literatur}

Antweiler C (2007) Was ist den Menschen gemeinsam? Über Kultur und Kulturen. Wissenschaftliche Buchgesellschaft Darmstadt

Bauer S, Schanda H, Karakula H, Rudaleviciene P, Okribelashvili N, Chaudhry HR, Idemudia I, Gscheider S, Ritter $\mathrm{K}$, Stompe T (2011) Culture and the prevalence of hallucinations in schizophrenia. Compr Psychiatry 52, 319-325

Bulayeva KB, Glatt S|, Bulayev OA, Pavlova TA, Tsuang MT (2007) Genome-wide linkage scan of schizophrenia: a cross-isolate study. Genomics 89, 167-177

Cavalli-Sforza LL, Menozzi P, Piazza A (1994) The history and geography of human genes. University Press Princeton Princeton

Chiao JY (Hrsg.) (2009) Cultural neuroscience: Cultural influences on brain functions. Elsevier Amsterdam Boston Heidelberg

Choudhury S, Kirmayer LI (2009) Cultural neuroscience and psychopathology: prospects for cultural psychiatry. In: Chiao JY (Hrsg.) Cultural neuroscience: Cultural influences on brain functions. 263-283. Elsevier Amsterdam Boston Heidelberg

Kitayama S, Cohen D (Hrsg.) (2007) Handbook of cultural psychology. The Guilford Press New York London

Lin K-M, Singh BS, Chiu E (2008) Ethno-Psychopharmacology. Cambridge University Press Cambridge

Luhmann N (1993-1999) Gesellschaftssystem und Semantik. Band 4. Suhrkamp Frankfurt am Main

Luhmann N (1998) Die Gesellschaft der Gesellschaft. Suhrkamp Frankfurt am Main

Luhmann N (1999) Kultur als historischer Begriff. In: Luhmann N Gesellschaftsstruktur und Semantik. Studien zur Wissenschaftssoziologie der modernen Gesellschaft. Band 4. 31-54. Suhrkamp Frankfurt am Main

Mackay-Sim A, Féron F, Eyles D, Burne T, McGrath I (2004) Schizophrenia, vitamin D, and brain development. Int Rev Neurobiol 59, 351-80

McGrath J, Saha S, Welham J, El Saadi 0, MacCauley C, Chant D (2004) A systematic review of the incidence of schizophrenia: the distribution of rates and the influence of sex, urbanicity, migrant status and methodology. BMC Med 2, 13

Parin P, Morgenthaler F, Parin-Matthey G (1963) Die Weißen denken zu viel. Fischer Frankfurt am Main

Pfeiffer W (1994) Transkulturelle Psychiatrie. Thieme Stuttgart

Ritter K, Chaudhry HR, Zitterl W, Aigner M, Stompe T (2010) Subjektive Krankheitsmodelle zwischen spezifischer Krankheitserfahrung und Kultur. Neuropsychiatr 24, 33-41

Ritter K, Stompe T (2011) Transkulturelle Psychiatrie - Möglichkeiten und Grenzen. Spektrum Psychiatrie 1, 15-19

Robertz FI, Wickenhäuser R (2010) Kriegerträume. Warum unsere Kinder zu Gewalttätern werden. Herbig München

Saha S, Chant D, Welham J, McGrath I (2005) A systematic review of the prevalence of schizophrenia. PLoS Med 2, e141

Schütz A, Luckmann T (1979) Strukturen der Lebenswelt. Suhrkamp Frankfurt am Main

Srubal I, Renn J, Wenzel U (Hrsg.) (2005) Kulturen vergleichen. Sozial- und kulturwissenschaftliche Grundlagen und Kontroversen. VS Verlag für Sozialwissenschaften Wiesbaden

Stompe T (2006) Future directions of transcultural psychiatry. WPA-TPS Conference Vienna, April 2006. WACP Newsletter 2, 48-51 
Stompe T (2008) Überlegungen zur Problematik kulturvergleichender psycho-pathologischer Untersuchungen am Beispiel der Schizophrenie. In: Golsabahi S, Heise T (Hrsg.) Von Gemeinsamkeiten und Unterschieden. Verlag für Wissenschaft und Bildung Berlin

Stompe T (2010) Transkulturelle Wahnforschung. Psychopraxis 13, 22-27

Stompe T, Ortwein-Swoboda G, Ritter K, Schanda H (2003) Old wine in new bottles? Stability and plasticity of the contents of schizophrenic delusions. Psychopathology 36, 6-12

Stompe T, Ritter K, Hauska S, Chaudhry HR (2010) Krankheitsmodelle bei Patienten mit Schizophrenie in Pakistan und in Österreich unter besonderer Berücksichtigung von Genderaspekten. Neuropsychiatr 24, 252-258

Stompe T, Ritter K, Schrank B (2010a) Depression und Kultur. Neuropsychiatr 24, 33-41

Tellenbach H (1960) Melancholie. Springer Berlin Heidelberg New York Tokio

Thomas A, Kinast E-U, Schroll-Machl S (2009) Handbuch Interkulturelle Kommunikation und Kooperation. Vandenhoeck u. Ruprecht Göttingen

Torrey F (2001) The invisible plague: the rise of mental illness from 1750 to the present. Rutgers University Press 